\title{
Ultra-thin ZnSe: Anisotropic and flexible crystal structure
}

\author{
C. Bacaksiz ${ }^{\mathrm{a}, *}$, R.T. Senger ${ }^{\mathrm{a}}$, H. Sahin ${ }^{\mathrm{b}}$ \\ a Department of Physics, Izmir Institute of Technology, 35430 Izmir, Turkey \\ ${ }^{\mathrm{b}}$ Department of Photonics, Izmir Institute of Technology, 35430 Izmir, Turkey
}

\section{A R T I C L E I N F O}

\section{Article history:}

Received 10 February 2017

Accepted 4 March 2017

Available online 7 March 2017

\section{Keywords:}

Ultra-thin two-dimensional

density-functional-theory

\begin{abstract}
A B S T R A C T
By performing density functional theory-based calculations, we investigate the structural, electronic, and mechanical properties of the thinnest ever ZnSe crystal [11]. The vibrational spectrum analysis reveals that the monolayer $\mathrm{ZnSe}$ is dynamically stable and has flexible nature with its soft phonon modes. In addition, a direct electronic band gap is found at the gamma point for the monolayer structure of ZnSe. We also elucidate that the monolayer $\mathrm{ZnSe}$ has angle dependent in-plane elastic parameters. In particular, the in-plane stiffness values are found to be 2.07 and $6.89 \mathrm{~N} / \mathrm{m}$ for the arm-chair and zig-zag directions, respectively. The angle dependency is also valid for the Poisson ratio of the monolayer ZnSe. More significantly, the in-plane stiffness of the monolayer ZnSe is the one-tenth of Young modulus of bulk zb-ZnSe which indicates that the monolayer $\mathrm{ZnSe}$ is a quite flexible single layer crystal. With its flexible nature and in-plane anisotropic mechanical properties, the monolayer $\mathrm{ZnSe}$ is a good candidate for nanoscale mechanical applications.
\end{abstract}

(c) 2017 Elsevier B.V. All rights reserved.

\section{Introduction}

The II-VI binary compounds are well-known and have been extensively studied semiconductor structures. Mostly II-VI structures crystallize in the zinc-blende $(z b)$ and wurtzite $(w z)$ phases, and depending on their size and shape, they exhibit diverse electronic and optical properties [1-4]. On the other hand, following the realization of one atom thick carbon crystal, graphene [5], the two-dimensional (2D) materials have attracted enormous interest and the 2D family has grown rapidly in the last decade.

Although, most of the ultra-thin crystals have layered structure in their bulk forms, there are several studies that revealed possibility of synthesis of 2D crystals from non-layered materials of II-VI binary compounds [6-17]. Among these studies, Sun et al. [11] reported the synthesis of monolayer ZnSe. According to their observations, the monolayer $\mathrm{ZnSe}$ is ultra-flexible and has a band gap of $\sim 3.5 \mathrm{eV}$. Motivated by this observations, several computational studies were also reported. Tong et al. investigated the various compounds having zinc-blende form, comparatively [18]. They predicted a phase of monolayer ZnSe resembling a slab of the rock-salt crystal ( $t-\mathrm{ZnSe})$ and claimed that this phase is energetically more favorable than that observed. In addition, Zhou et al. compared different monolayer phases of ZnSe by means of many

\footnotetext{
* Corresponding author.

E-mail address: cihanbacaksiz@iyte.edu.tr (C. Bacaksiz).
}

body perturbation theory on top of density functional theory [19]. They also predicted that the $\mathrm{t}-\mathrm{ZnSe}$ phase is dynamically stable and energetically favorable. More recently, Zhou et al. predicted the structural transition from the experimentally observed phase to the $\mathrm{t}-\mathrm{ZnSe}$ phase under lateral pressure at room temperature by using molecular dynamic simulations [20].

Although, the computational studies asserted that the synthesized monolayer ZnSe is meta-stable, the dynamical stability and also the mechanical properties are still unknown. Here, using first principles calculations based on density functional theory (DFT), we investigate structural, electronic, and mechanical properties of the synthesized monolayer ZnSe. Our investigation reveals that the monolayer ZnSe is dynamically stable and possesses angle dependent elastic parameters such as in-plane stiffness and Poisson ratio.

The paper is organized as follows: in Section 2 we give details of our computational methodology. An overview of the structural phases and the electronic properties of monolayer ZnSe are presented in Section 3. In Section 4 we focus on the mechanical properties of ZnSe. Finally, the conclusion of the study is given in Section 5.

\section{Computational methodology}

We performed density functional theory (DFT) based calculations to investigate the structural, electronic, and mechanical properties of the monolayer ZnSe by using the Vienna ab-initio simulation package, VASP [21-23]. To describe electron exchange 

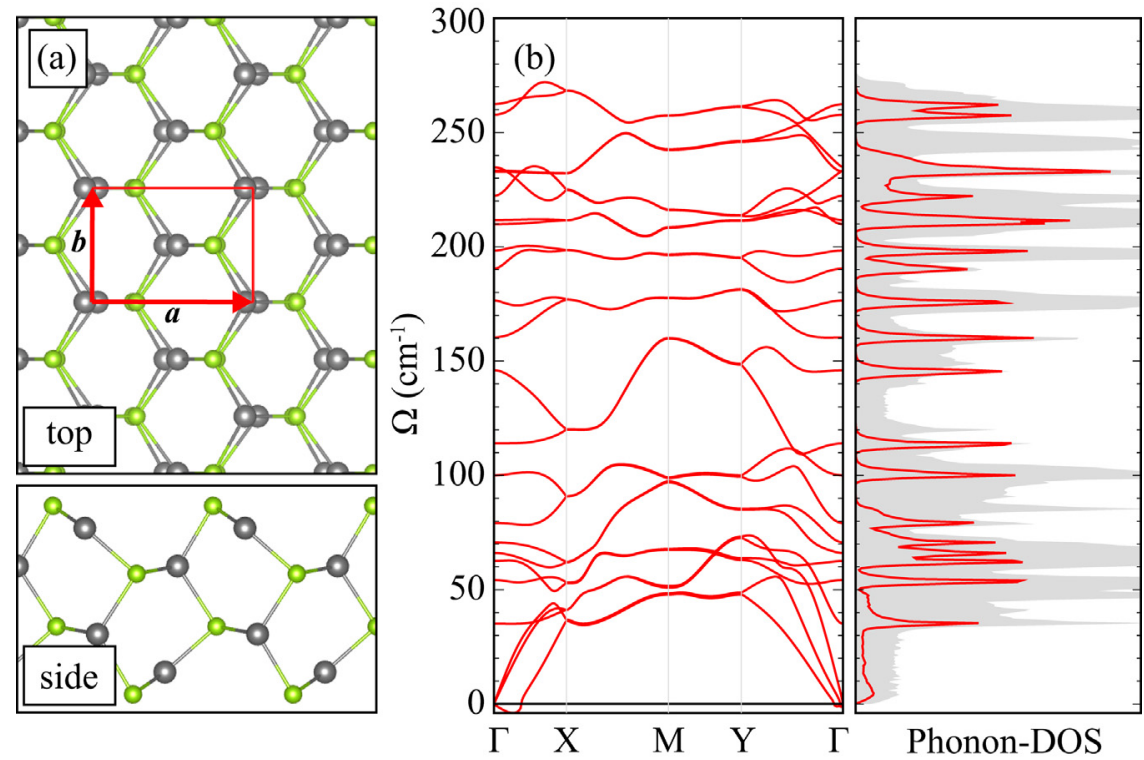

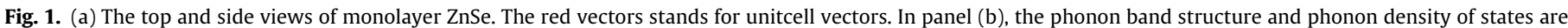

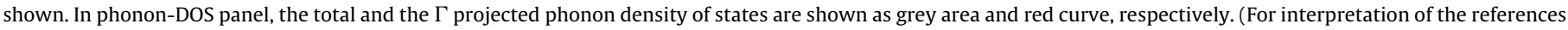
to color in this figure legend, the reader is referred to the web version of this article.)

and correlation, the Perdew-Burke-Ernzerhof (PBE) form of the generalized gradient approximation (GGA) [24] was adopted. The vdW forces were taken into account by using the DFT-D2 method of Grimme [25,26]. The vibrational properties were calculated for 128-atom-supercell using the small displacement methodology [27]. To obtain the charge distribution of the configurations, a Bader charge analysis was used [28,29].

The analyses were carried out by using following parameters. The total energy difference between the sequential steps in the iterations was taken $10^{-5} \mathrm{eV}$ as convergence criterion. The convergence for the Hellmann-Feynman forces on each unitcell was taken to be $10^{-4} \mathrm{eV} / \mathrm{A}$. $0.05 \mathrm{eV}$ of Gaussian smearing factor was used and the pressures on the unit cell were decreased to a value less then $1.0 \mathrm{kB}$ in all three directions. For the determination of accurate charge densities, Brillouin zone integration was performed using a $20 \times 30 \times 1 \Gamma$-centered mesh for the primitive unit cell. To avoid interactions between adjacent monolayer $\mathrm{ZnSe}$, our calculations were performed with a large unit cell including $15 \AA$ Aacuum space.

\section{Structural and electronic properties}

In this section, we investigate the structural and electronic properties of the monolayer ZnSe. As the experimental observation [11] of 4-atom-thick monolayer ZnSe, we truncate $4 \mathrm{Zn}$-Se sublayers from the bulk $z b$-ZnSe for the initial configuration of the DFT-based structural optimization. As shown in Fig. 1(a), a rectangular unitcell with 8 atoms is constructed and the lattice parameters are found to be $a=5.57$ and $b=3.96 \AA$ as given in Table 1 . At the optimized surfaces of the monolayer $\mathrm{ZnSe}$, the outermost $\mathrm{Zn}$ and Se atoms are distorted as shown in the side view of Fig. 1(a). The outermost $\mathrm{Zn}$ atoms have three coordinating Se atoms with the same bond length of $2.40 \AA$. The inner $\mathrm{Zn}$ atoms have 4 coordinating Se atoms with a tetrahedral type as in bulk crystal but the bong lengths vary between 2.43 and $2.50 \AA$. These bond lengths are not significantly different from the bulk value of $2.45 \AA$. The Bader charge analysis reveals that slightly varying bonds exhibit the same charge depletion of $0.7 e^{-}$from $\mathrm{Zn}$ to $\mathrm{S}$. In addition, the cohesive energy is found to $2.69 \mathrm{eV}$ which slightly differ from the bulk value of $2.84 \mathrm{eV}$ per atom.

After the structural optimization, the dynamical stability of the monolayer ZnSe is also examined. As shown in Fig. 1(b), the phonon-band dispersion reveals that the monolayer ZnSe is dynamically stable. In addition, the maximum frequency which corresponds the optical phonon mode appears at $262 \mathrm{~cm}^{-1}$. The low frequencies of the vibrational modes indicates the flexibility of the monolayer ZnSe. The total and the $\Gamma$ projected phonon density of states are shown in Fig. 1(b) so that a possible Raman measurement of a monolayer $\mathrm{ZnSe}$ can be compared.

In addition, the energy band gap of monolayer ZnSe is calculated to be $1.91 \mathrm{eV}$ within GGA + SOC. As shown in Fig. 2(a), the valence band maximum (VBM) and conduction band minimum (CBM) appears at the $\Gamma$ point. For comparison, using the same methodology, the band gap of bulk $z b$-ZnSe is also calculated to be $1.32 \mathrm{eV}$ which is $\sim 0.6 \mathrm{eV}$ lower than that of monolayer. Note that the bulk $z b-\mathrm{ZnSe}$ is known to have direct the band gap of $\sim 2.8 \mathrm{eV}$ [30] and the band gap of the monolayer is reported to be $3.5 \mathrm{eV}[11]$. Although the calculated band gap values are underestimated, the difference between the bulk and monolayer is consistent with the experimental difference of $\sim 0.7 \mathrm{eV}$.

We also calculated the electronic density of state which is shown in Fig. 2(b). The VBM is dominated by the Se atoms. In the CBM, it is seen that the $\mathrm{Zn}$ and Se atoms contribute. Since the outermost atoms have different bond coordinations, more detailed analysis is needed. Therefore, we focus on the band edges by calculating

Table 1

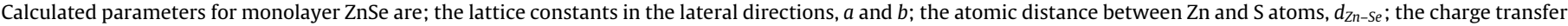
from $\mathrm{Zn}$ to Se atom, $\Delta \rho$; and the cohesive energy per atom, $E_{c}$. $E_{g}^{\mathrm{GGA}+\mathrm{SOC}}$ is the band gap values within GGA+SOC.

\begin{tabular}{|c|c|c|c|c|c|c|}
\hline & $a(\AA)$ & $b(\AA)$ & $d_{Z n-S e}(\AA)$ & $\Delta \rho\left(e^{-}\right)$ & $E_{c}(\mathrm{eV})$ & $E_{g}^{\mathrm{GGA}+\mathrm{SOC}}(\mathrm{eV})$ \\
\hline Monolayer & 5.57 & 3.96 & $2.40-2.50$ & 0.7 & 2.69 & 1.88 \\
\hline$z b$-bulk & 5.65 & - & 2.45 & 0.7 & 2.84 & 1.32 \\
\hline
\end{tabular}



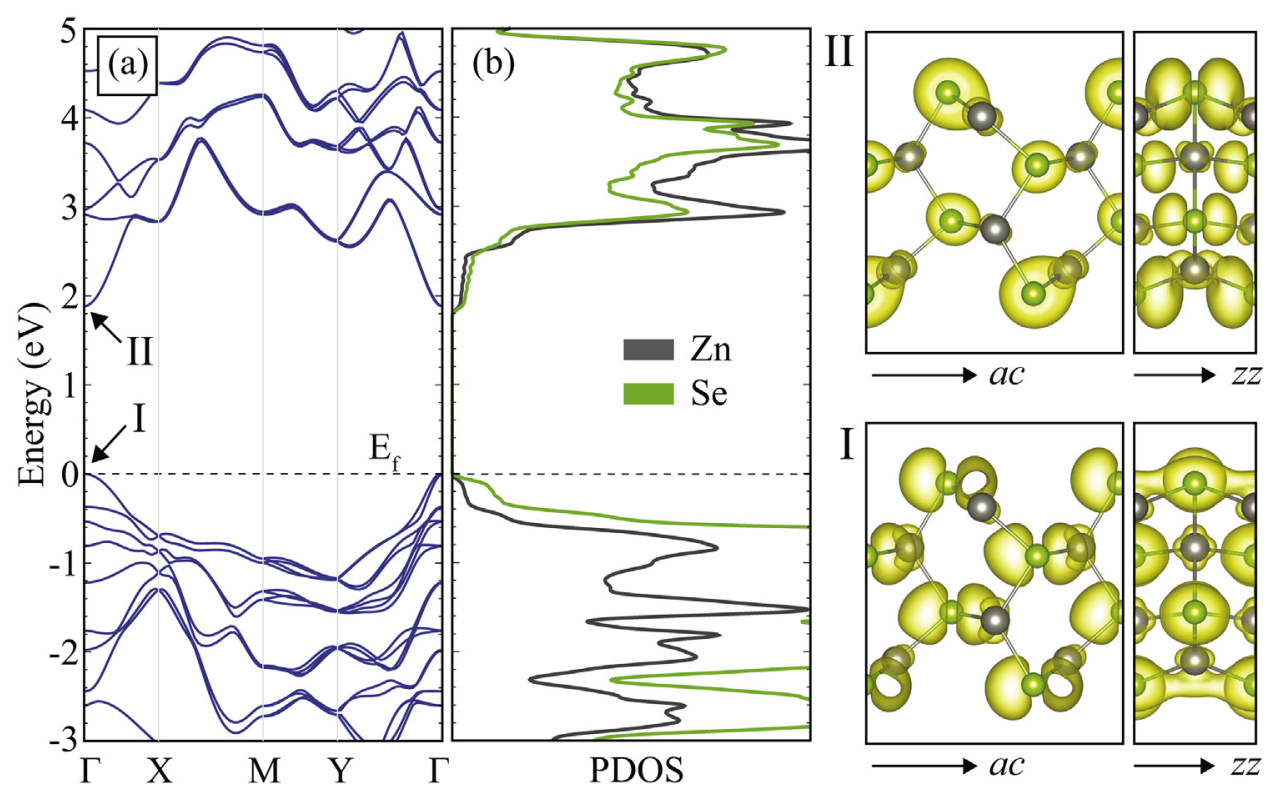

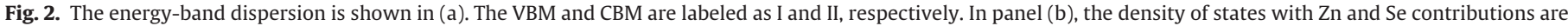

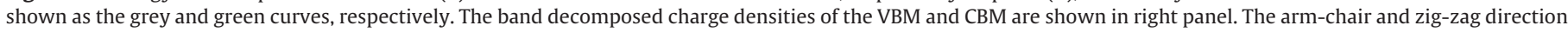
are represented by $a c$ and $z z$, respectively. (For interpretation of the references to color in this figure legend, the reader is referred to the web version of this article.)

the band decomposed charge densities of the VBM and CBM. As shown in the right panel of Fig. 2, the VBM(I) is dominated by the $p_{x}$ orbitals of Se atoms, on the other hand, the CBM (II) is dominated by the $p_{y}$ orbitals Se atoms. For both band edges, the charge densities of the inner atoms are more localized. The outer Se and $\mathrm{Zn}$ charge densities extend over the surface of the monolayer especially along the zig-zag $(y)$ direction.

\section{Mechanical properties}

The dimensional reduction from bulk to monolayer brings not only novel electronic properties but also some unusual mechanical properties. The flexibility of the monolayer $\mathrm{ZnSe}$ is emphasized by Sun et al. [11] however it is still needed to be elucidated in terms of elastic parameters. Therefore, in this section, we investigate the mechanical properties of the monolayer $\mathrm{ZnSe}$. To obtain the response of the monolayer to the in-plane strain, the total energy differences are calculated under the combinations of strain values along the arm-chair and zig-zag directions as shown in Fig. 3(a). We restrict our calculation in elastic region in which the strains are considered to be between $\mp 2 \%$ in each direction. As it is seen, the maximum energy increase is obtained under biaxial strain. Moreover, the change in the energy is larger when the structure strained along the arm-chair $(x)$ direction as compared to that of the zigzag $(y)$ direction. Since there is remarkable difference between the strain directions, which is a direct indication of the structural anisotropy, the energy difference is also calculated for the applied strain along the specific direction having an angle $\theta$ to the armchair $(x)$ direction, so that the angle dependent elastic parameters can be depicted.

In Fig. 3(b) and (c), the tensile and compressive straindependent energy curves are shown separately. As it is seen, the response to the strains are not circular but varying with the angle. The maximum energy differences of 92 and $91 \mathrm{meV}$ are obtained when the system strained along the direction of $30^{\circ}$ and $90^{\circ}$ (zigzag) angle with respect to the arm-chair direction, respectively. The minimum energy difference (a few $\mathrm{meV}$ ), on the other hand, is obtained at the angle of $0^{\circ}$ and $60^{\circ}$. The response in the level of meV indicates the flexibility of the monolayer ZnSe. Using the strain-energy relation, we further investigate the in-plane stiffness and the Poisson ratio with considering the angle dependency.

The relation between strain and the total energy difference is considered to be obey the formula of $E_{s}=c_{1} \varepsilon_{\theta}^{2}+c_{2} \varepsilon_{\phi}^{2}+c_{3} \varepsilon_{\theta} \varepsilon_{\phi}$, where the $\varepsilon_{\theta}$ and $\varepsilon_{\phi}$ applied strain values along the corresponding direction and the direction which is perpendicular to $\theta\left(\phi-\theta=90^{\circ}\right)$, respectively. The coefficients of $c_{1}, c_{2}$, and $c_{3}$ are obtained separately by fitting the energy-strain equation given above. Since the monolayer ZnSe possesses anisotropic response depending on the strain direction, the $c_{1}$ and $c_{2}$ are not equal. By using the coefficients of $c_{1}, c_{2}$, and $c_{3}$ the elastic parameters such as in-plane stiffness and Poisson ratio can be obtained.

\subsection{In-plane stiffness}

The stiffness defines the resistivity of a material to the structural deformation under mechanical force. It is one of the fundamental properties that describes the flexibility or rigidity of a material. To reveal the mechanical properties, we investigate the in-plane stiffness by using the relation of $C_{\theta}=\left[4 c_{1} c_{2}-\left(c_{3}^{2} / 2 c_{2}\right)\right] / A_{0}$, where the $c_{1}, c_{2}$, and $c_{3}$ are the coefficients that are determined by the energystrain relation given above, and $A_{0}$ is the area of the unstrained supercell.

The calculated in-plane stiffness, $C_{\theta}$, is shown as a polar plot in Fig. 3(d). As a consequence of the angle dependent strain energy, the in-plane stiffness values also vary between 1.0 and $8.0 \mathrm{~N} / \mathrm{m}$ depending on the strain direction. The in-plane stiffness values for the arm-chair and zig-zag directions are found to be $C_{0}=6.89$ and $C_{90}=2.07 \mathrm{~N} / \mathrm{m}$, respectively. In addition, the maximum and minimum values are obtained for the angles of $30^{\circ}$ and $75^{\circ}$, respectively.

Here, the calculated stiffness can be compared with the Young's modulus of the bulk $z b$-ZnSe yet differing from the bulk form, the thickness of the monolayer ZnSe is considered when the in-plane stiffness is obtained. Therefore, we divide the calculated in-plane stiffness by the thickness of the monolayer $\mathrm{ZnSe}$ and these effective values in the arm-chair and zig-zag directions are found to be $3.2 \times 10^{9}$ and $10.5 \times 10^{9} \mathrm{~N} / \mathrm{m}^{2}$. The Young's modulus of the bulk 
(a)

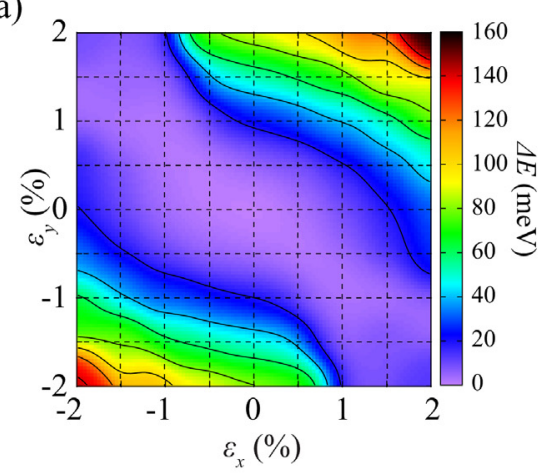

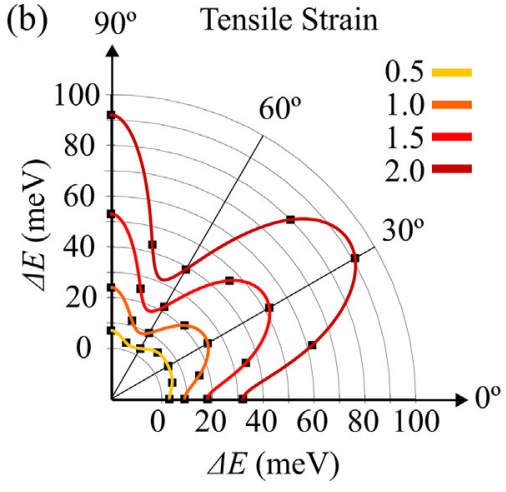

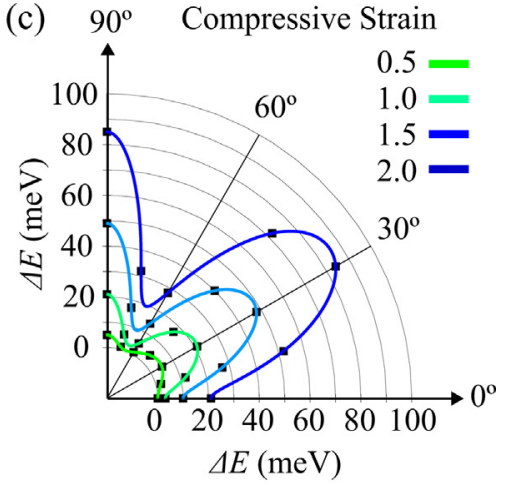

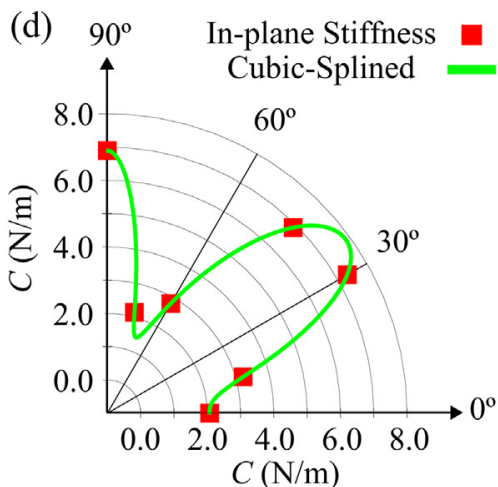

(e)

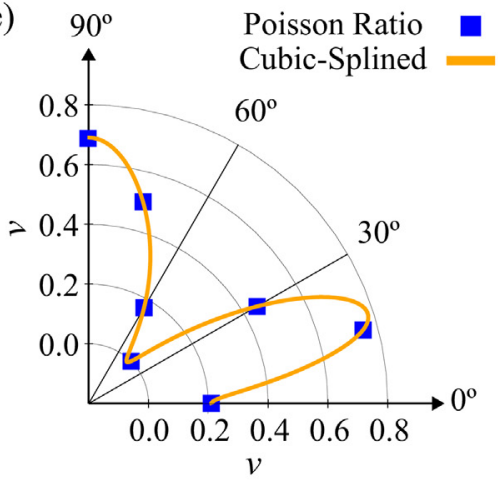

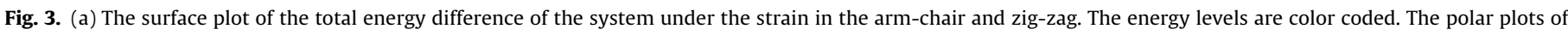

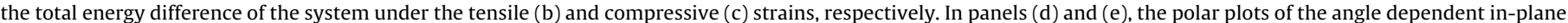
stiffness and the Poisson ratio are shown, respectively.

$z b$-ZnSe is known to be $67.2 \times 10^{9} \mathrm{~N} / \mathrm{m}^{2}$. As it is seen, the there is a dramatic decrease in the stiffness when the dimensionality of structure reduces from 3D to 2D. The small in-plane stiffness values confirmed the flexibility of the monolayer ZnSe which is reported by Sun et al. [11]. In addition, as compared to calculated values of 91 and 77 N/m for GaS and GaSe [31], respectively, the in-plane stiffness of the monolayer $\mathrm{ZnSe}$ is also very low.

\subsection{Poisson ration}

One of the important fundamental elastic constants of a material is the Poisson ratio. Briefly, when a material is stretched (compressed) along one particular direction, it usually shrinks (expands) in the perpendicular direction. The amount of that kind of deformations are governed by the Poisson ratio which must be investigated for well-identification of the mechanical properties. Therefore, we calculated the angle dependent Poisson ratio of the monolayer $\mathrm{ZnSe}$ by using the relation of $v_{\theta}=c_{3} / 2 c_{1}$, where the $c_{1}$ and $c_{3}$ are the coefficients introduced above.

The polar plot of the calculated Poisson ratio, $v_{\theta}$ is shown in Fig. 3(e). Apparently, the angle dependency of the Poisson ratio differs from that of in-plane stiffness. The values vary between 0.0 and 0.75 . The maximum Poisson ratio is obtained when the directional angle with of the strain is $15^{\circ}$. For the arm-chair and zig-zag directions, the Poisson ratio are $v_{0}=0.21$ and $v_{90}=0.69$, respectively. More significantly, the Poisson ratio for the angle of 45 is about 0.0 . When we compare the calculated Poisson ratio of the monolayer $\mathrm{ZnSe}$ with that of bulk $z b-\mathrm{ZnSe}$, which is known to be 0.28 , values are not compatible. Since the monolayer $\mathrm{ZnSe}$ is very flexible, the Poisson ratio values given above must be considered qualitatively. Therefore, one can conclude that the in-plane anisotropy is still valid for the Poisson ratio of the monolayer ZnSe.

\section{Conclusions}

In this study, we investigate the synthesized monolayer ZnSe by performing state-of-the-art first principles calculations. The structural optimization reveals that outermost $\mathrm{Zn}$ and Se atoms relaxed in a distorted form and the inner $\mathrm{Zn}$ and Se atoms have tetrahedral bond coordinations. The phonon analysis shows that the monolayer $\mathrm{ZnSe}$ is dynamically stable. In addition, the monolayer, ZnSe has a direct electronic band gap at the $\Gamma$ symmetry point. The electronic density of states analysis shows that VBM and CBM are dominated by the $p_{x}$ and $p_{y}$ orbitals of Se atoms, respectively. Furthermore, we investigate the mechanical properties of the monolayer $\mathrm{ZnSe}$ in the elastic region. First of all, the energy-strain calculation shows that the monolayer ZnSe has anisotropic response which depends to the direction of the strain. In particular, the in-plane stiffness values are found to be 6.89 and $2.07 \mathrm{~N} / \mathrm{m}$ for the arm-chair and zig-zag directions, respectively, which are effectively very low as compared to the Young's modulus of the bulk $z b$-ZnSe. The low in-plane stiffness values are also confirmed the experimental observation of the flexibility of monolayer ZnSe. Moreover, the Poisson ratio is calculated to be 0.21 and 0.69 for the arm-chair and zig-zag directions, respectively. With its flexibility nature and in-plane anisotropic mechanical properties, the monolayer ZnSe appears quite suitable for flexible nanoscale device applications.

\section{Acknowledgements}

The calculations were performed at TUBITAK ULAKBIM, High Performance and Grid Computing Center (TR-Grid eInfrastructure). CB and RTS acknowledge the support from TUBITAK Project No. 114F397. HS acknowledges support from Bilim Akademisi - The Science Academy, Turkey under the BAGEP program. HS acknowledges financial support from the Scientific and 

Technological Research Council of Turkey (TUBITAK) under the
project number 116 C073.

\section{References}

[1] C. Murray, D.J. Norris, M.G. Bawendi, Synthesis and characterization of nearly monodisperse CdE ( $E$ = sulfur, selenium, tellurium) semiconductor nanocrystallites, J. Am. Chem. Soc. 115 (19) (1993) 8706-8715.

[2] L. Manna, D.J. Milliron, A. Meisel, E.C. Scher, A.P. Alivisatos, Controlled growth of tetrapod-branched inorganic nanocrystals, Nat. Mater. 2 (6) (2003) 382-385.

[3] Y. Yin, A.P. Alivisatos, Colloidal nanocrystal synthesis and the organic inorganic interface, Nature 437 (7059) (2005) 664-670.

[4] X. Peng, L. Manna, W. Yang, J. Wickham, E. Scher, A. Kadavanich, A.P. Alivisatos, Shape control of CdSe nanocrystals, Nature 404 (6773) (2000) 59-61.

[5] K.S. Novoselov, A.K. Geim, S.V. Morozov, D. Jiang, Y. Zhang, S.V. Dubonos, I.V. Grigorieva, A.A. Firsov, Electric field effect in atomically thin carbon films, Science 306 (5696) (2004) 666-669.

[6] S. Ithurria, B. Dubertret, Quasi 2D colloidal CdSe platelets with thicknesses controlled at the atomic level, J. Am. Chem. Soc. 130 (49) (2008) 16504-16505.

[7] J.S. Son, X.D. Wen, J. Joo, J. Chae, S.I. Baek, K. Park, J.H. Kim, K. An, J.H. Yu, S.G. Kwon, S.H. Choi, Z.W. Wang, Y.W. Kim, Y. Kuk, R. Hoffmann, T. Hyeon, Large scale soft colloidal template synthesis of $1.4 \mathrm{~nm}$ thick CdSe nanosheets, Angew. Chem. 121 (37) (2009) 6993-6996.

[8] S. Ithurria, G. Bousquet, B. Dubertret, Continuous transition from 3D to 1D confinement observed during the formation of CdSe nanoplatelets, J. Am. Chem. Soc. 133 (9) (2011) 3070-3077.

[9] Z. Li, X. Peng, Size/shape-controlled synthesis of colloidal CdSe quantum disks: ligand and temperature effects, J. Am. Chem. Soc. 133 (17) (2011) 6578-6586.

[10] S. Ithurria, M.D. Tessier, B. Mahler, R.P S.M. Lobo, B. Dubertret, A.L. Efros, Colloidal nanoplatelets with two-dimensional electronic structure, Nat. Mater. 10 (12) (2011) 936-941.

[11] Y. Sun, Z. Sun, S. Gao, H. Cheng, Q. Liu, J. Piao, T. Yao, C. Wu, S. Hu, S. Wei, Y. Xie, Fabrication of flexible and freestanding zinc chalcogenide single layers, Nat. Commun. 3 (2012) 1057.

[12] H. Park, H. Chung, W. Kim, Synthesis of ultrathin wurtzite ZnSe nanosheets, Mater. Lett. 99 (2013) 172-175.

[13] Y. Sun, Q. Liu, S. Gao, H. Cheng, F. Lei, Z. Sun, Y. Jiang, H. Su, S. Wei, Y. Xie, Pits confined in ultrathin cerium (IV) oxide for studying catalytic centers in carbon monoxide oxidation, Nat. Commun. 4 (2013) 2899.
[14] Y. Sun, F. Lei, S. Gao, B. Pan, J. Zhou, Y. Xie, Atomically thin tin dioxide sheets for efficient catalytic oxidation of carbon monoxide, Angew. Chem. Int. Ed. 52 (40) (2013) 10569-10572.

[15] X. Zhang, J. Zhang, J. Zhao, B. Pan, M. Kong, J. Chen, Y. Xie, Half-metallic ferromagnetism in synthetic $\mathrm{Co}_{9} \mathrm{Se}_{8}$ nanosheets with atomic thickness, J. Am. Chem. Soc. 134 (29) (2012) 11908-11911.

[16] X. Zhang, Q. Liu, L. Meng, H. Wang, W. Bi, Y. Peng, T. Yao, S. Wei, Y. Xie, In-plane coassembly route to atomically thick inorganic organic hybrid nanosheets, ACS Nano 7 (2) (2013) 1682-1688.

[17] F. Lei, Y. Sun, K. Liu, S. Gao, L. Liang, B. Pan, Y. Xie, Oxygen vacancies confined in ultrathin indium oxide porous sheets for promoted visible-light water splitting, J. Am. Chem. Soc. 136 (19) (2014) 6826-6829.

[18] C.J. Tong, H. Zhang, Y.N. Zhang, H. Liu, L.M. Liu, New manifold two-dimensional single-layer structures of zinc-blende compounds, J. Mater. Chem. A 2 (42) (2014) 17971-17978.

[19] J. Zhou, B.G. Sumpter, P.R. Kent, J. Huang, A novel and functional single-layer sheet of ZnSe, ACS Appl. Mater. Interfaces 7 (3) (2015) 1458-1464.

[20] L. Li, P. Li, N. Lu, J. Dai, X.C. Zeng, Tunable bandgaps: simulation evidence of hexagonal to tetragonal ZnSe structure transition: a monolayer material with a wide range tunable direct bandgap, Adv. Sci. 2 (12) (2015) 1500290.

[21] G. Kresse, J. Hafner, Ab initio molecular dynamics for liquid metals, Phys. Rev. B 47 (1) (1993) 558.

[22] G. Kresse, J. Furthmller, Efficient iterative schemes for ab initio total-energy calculations using a plane-wave basis set, Phys. Rev. B 54 (16) (1996) 11169.

[23] G. Kresse, D. Joubert, From ultrasoft pseudopotentials to the projector augmented-wave method, Phys. Rev. B 59 (3) (1999) 1758.

[24] J.P. Perdew, K. Burke, M. Ernzerhof, Generalized gradient approximation made simple, Phys. Rev. Lett. 77 (18) (1996) 3865.

[25] S. Grimme, Semiempirical GGAtype density functional constructed with a longrange dispersion correction, J. Comput. Chem. 27 (15) (2006) 1787-1799.

[26] T. Bucko, J. Hafner, S. Lebegue, J.G. Angyn, Improved description of the structure of molecular and layered crystals: ab initio DFT calculations with van der Waals corrections, J. Phys. Chem. A 114 (43) (2010) 11814-11824.

[27] D. Alfe, PHON: a program to calculate phonons using the small displacement method, Comput. Phys. Commun. 180 (12) (2009) 2622-2633.

[28] G. Henkelman, A. Arnaldsson, H. Jonsson, A fast and robust algorithm for Bader decomposition of charge density, Comput. Mater. Sci. 36 (3) (2006) 354-360.

[29] R.F.W. Bader, Atoms in Molecules A Quantum Theory, Oxford University Press, Oxford, UK, 1990.

[30] A.D. Yoffe, Low-dimensional systems: quantum size effects and electronic properties of semiconductor microcrystallites (zero-dimensional systems) and some quasi-two-dimensional systems, Adv. Phys. 42 (2) (1993) 173-262.

[31] M. Yagmurcukardes, R.T. Senger, F.M. Peeters, H. Sahin, Mechanical properties of monolayer GaS and GaSe crystals, Phys. Rev. B 94 (24) (2016) 245407. 